\title{
Visual Analysis of One-to-Many Matched Graphs ${ }^{\star}$
}

\author{
Emilio Di Giacomo, Walter Didimo, Giuseppe Liotta, and Pietro Palladino \\ Dipartimento di Ingegneria Elettronica e dell'Informazione, \\ Università degli Studi di Perugia, Italy \\ \{digiacomo, didimo, liotta, palladino\}@diei.unipg.it
}

\begin{abstract}
Motivated by applications of social network analysis and of Websearch clustering engines, we describe an algorithm and a system for the display and the visual analysis of two graphs $G_{1}$ and $G_{2}$ such that each $G_{i}$ is defined on a different data set with its own primary relationships and there are secondary relationships between the vertices of $G_{1}$ and those of $G_{2}$. Our main goal is to compute a drawing of $G_{1}$ and $G_{2}$ that makes clearly visible the relations between the two graphs by avoiding their crossings, and that also takes into account some other important aesthetic requirements like number of bends, area, and aspect ratio. Application examples and experiments on the system performances are also presented.
\end{abstract}

\section{Introduction}

The visual analysis of complex data sets is one of the most natural applications of graph drawing technologies (see, e.g., [2-4]). A typical application scenario consists of a set of data (nodes) and one or more relationships among these data (each relationship is a set of edges); therefore one is given one or more graphs on the same set of nodes. Both each graph must be visualized in a readable way and possible similarities among the different graphs must be easily detected by looking at the different drawings. This scenario has, for example, motivated a rich body of papers and systems about simultaneous graph embeddings and visualizations of evolving graphs (see, e.g., [7, 13-16]).

Recently, Collins and Carpendale [8] proposed a new research direction devoted to the visual comparison and analysis of heterogeneous data sets. The input consists of $n$ sets of data $D_{1}, D_{2}, \ldots, D_{n}$, such that for each $D_{i}$ a distinct set of primary relationships (i.e., a distinct graph) is defined; also, there are secondary relationships which model semantic connections between data belonging to different sets. The visualization consists of a set of $n$ drawings (one for each graph) on top of which the edges that represent the secondary relationships are displayed. Collins and Carpendale present a system, called VISLINK, where each graph is drawn on a distinct plane and the secondary relationships are links between these planes (see Fig. 1(a) for a schematic illustration). The work by Collins and Carpendale extends a previous work by Schneiderman and Aris where multi-plane views with inter-plane edges are used to visualize different semantic substrates of a same graph [19](see Fig. 1(b) for an illustration).

\footnotetext{
* Research partially supported by the MIUR Project "MAInSTREAM: Algorithms for Massive Information Structures and Data Streams".
} 


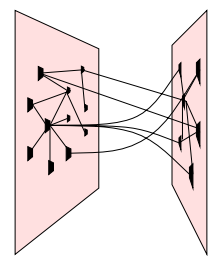

(a)

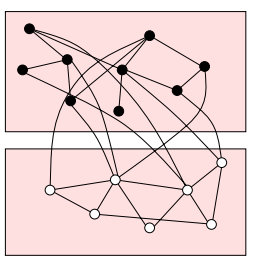

(b)

Fig. 1. Schematic illustrations of a visualization (a) adopted by VisLink, (b) using different semantic substrates of a same network. In both the visualizations the drawing on each plane has been computed without taking into account the relationships with the other. This may cause many crossings between inter-sets relationships.

Motivated by applications of social network analysis and of Web search clustering engines, we elaborate on the concepts by Collins and Carpendale by studying the following problem: We are given two graphs $G_{1}$ and $G_{2}$ and a function that defines a set of secondary relationships by mapping some of the vertices of $G_{1}$ to some other vertices of $G_{2}$; we aim at visually analyzing and interacting both with $G_{1}, G_{2}$ and with their secondary relationships. We observe that the systems described in $[8,19]$ follow the common approach of drawing each graph independently of each other. As a result, the secondary edges may be difficult to read as they can have many crossings. Our main goal is to design a system where the two drawings are computed by taking into account the edge-crossing minimization of the secondary edges. We focus on one-to many relationships between $G_{1}$ and $G_{2}$, i.e., vertices of $G_{1}$ are associated with disjoint subsets of vertices of $G_{2}$. The main contributions of the paper are the following:

- We introduce the concept of one-to-many matched graphs and define drawing conventions for these graphs in a strong and non-strong model. Both drawings require the secondary relationships between the graphs not to cross each other (Sect. 2).

- We describe a system that computes strong and non-strong one-to-many matched drawings of the input graphs by also taking into account the optimization of important aesthetic requirements. Furthermore, the system provides the user with several interaction functionalities that make it possible to analyze the drawings at different levels of details by collapsing/expanding clusters and by filtering information with the definition of node/edge thresholds (Sect. 3). Our drawing approach combines orthogonal drawings in the topology driven approach with circular drawing algorithms, and adopts an edge bundling technique to reduce the visual complexity introduced by some links.

- We show the effectiveness of the system by presenting application examples (Sect. 4), and an experimental study on the system performances (Sect. 5).

We finally remark that the problem of drawing two matched planar graphs $G_{1}$ and $G_{2}$ with one-to-one secondary relationships between them have been originally studied in [11], where it is required that the drawing of each $G_{i}$ is planar and that the secondary edges are represented as non-intersecting horizontal segments. 


\section{One-to-Many Matched Graphs and Drawings}

We assume familiarity with basic concepts of graph planarity and graph drawing [10]. If $G$ is a graph, we denote by $\Gamma(G)$ a drawing of $G . \Gamma(G)$ is an orthogonal drawing if each edge is drawn as a chain of horizontal and vertical segments. A bend in $\Gamma(G)$ is a point of an edge shared by a horizontal and a vertical segment of the edge. A drawing $\Gamma(G)$ is a circular drawing if there is a circle passing through all vertices and each edge is drawn as a straight-line segment. In the following, if $G=(V, E)$ is a graph and $V^{\prime} \subseteq V$ we denote by $G\left(V^{\prime}\right)$ the subgraph of $G$ induced by the vertices of $V^{\prime}$.

Let $G_{1}=\left(V_{1}, E_{1}\right)$ and $G_{2}=\left(V_{2}, E_{2}\right)$ be two distinct graphs. We say that $\left\langle G_{1}, G_{2}\right\rangle$ is a pair of one-to-many matched graphs if: $(i)$ Each vertex $u$ of $G_{1}$ is associated with a subset $M(u)=\left\{v_{1}, v_{2}, \ldots, v_{k}\right\}$ of vertices of $G_{2}$, which we call the cluster of $u$ in $G_{2}$; (ii) the set of clusters $\left\{M(u) \subseteq V_{2}: u \in V_{1}\right\}$ is a partition of $V_{2}$, i.e., $\bigcup_{u \in V_{1}} M(u)=$ $V_{2}$ and $\bigcap_{u \in V_{1}} M(u)=\emptyset$.

Let $\left\langle G_{1}, G_{2}\right\rangle$ be a pair of one-to-many matched graphs, and let $\Gamma\left(G_{1}\right), \Gamma\left(G_{2}\right)$ be drawings of $G_{1}$ and $G_{2}$, respectively. We say that $\left\langle\Gamma\left(G_{1}\right), \Gamma\left(G_{2}\right)\right\rangle$ is a one-to-many matched drawing if the following properties hold: (P1) The bounding boxes of $\Gamma\left(G_{1}\right)$ and $\Gamma\left(G_{2}\right)$ do not intersect. (P2) For each vertex $u$ of $G_{1}$, cluster $M(u)$ in $\Gamma\left(G_{2}\right)$ is bounded by a rectangular region $R(u)$ such that: $(i) G(M(u))$ is completely contained in $R(u)$; $($ ii $)$ each vertex $v \in V_{2} \backslash M(u)$ is outside $R(u)$; (iii) each edge of $G_{2}$ intersects the boundary of $R(u)$ at most once. (P3) For each vertex $u$ of $G_{1}$, there exists a simple curve $\ell(u)$ that connects the geometric shape $p_{u}$ representing $u$ in $\Gamma\left(G_{1}\right)$ to the boundary of $R(u)$ in $\Gamma\left(G_{2}\right)$, in such a way that $\bigcap_{u \in V_{1}} \ell(u)=\emptyset$.

In the paper, simple curves $\ell(u)$ are referred to as matching connections. Property (P3) guarantees that there is no intersection between distinct matching connections. A one-to-many matched drawing is said to be strong if the centers of the vertices of $\Gamma\left(G_{1}\right)$ have distinct $y$-coordinates and regions $R(u)$ are vertically ordered in $\Gamma\left(G_{2}\right)$ according to the positions of the corresponding vertices in $\Gamma\left(G_{1}\right)$. More formally, if $u_{1}, u_{2} \in V_{1}$ and $p_{u_{1}}$ is above $p_{u_{2}}$ in $\Gamma\left(G_{1}\right)$, then $R\left(u_{1}\right)$ is completely above $R\left(u_{2}\right)$ in $\Gamma\left(G_{2}\right)$. In the paper, a one-to-many matched drawing that is not strong will be referred to as a non-strong one-to-many matched drawing. Figure 2 shows two examples of oneto-many matched drawings for the same pair of graphs. The one in Fig. 2(b) is a strong one-to-many matched drawing.

\section{The System MOM}

In this section we present a system for the display and the visual analysis of one-tomany matched drawings. We call our system $\operatorname{MOM}^{1}$. Let $\left\langle G_{1}, G_{2}\right\rangle$ be a pair of oneto-many matched graphs to be visualized. MOM displays the drawing of $G_{1}$ to the left of the drawing of $G_{2}$, according to the following main criteria: (C1) It assumes that a drawing $\Gamma\left(G_{1}\right)$ is given as part of the input or that it can be computed using some classical graph drawing algorithm. (C2) It concentrates on the computation of $\Gamma\left(G_{2}\right)$, while trying to optimize a certain number of aesthetic criteria, other than guaranteeing that $\left\langle\Gamma\left(G_{1}\right), \Gamma\left(G_{2}\right)\right\rangle$ is a one-to-many matched drawing. (C3) Once $\Gamma\left(G_{2}\right)$ has

\footnotetext{
${ }^{1}$ MOM stands for Matched One-to-Many graphs.
} 


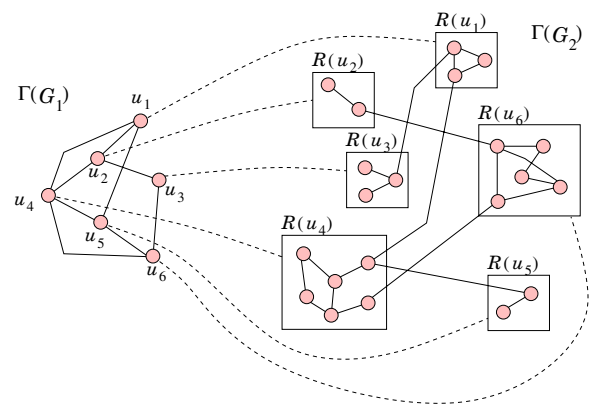

(a)

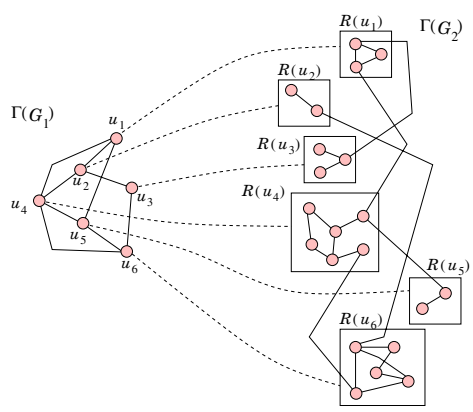

(b)

Fig. 2. (a) A (non-strong) one-to-many matched drawing of a pair of matched graphs. (b) A strong one-to-many matched drawing for the same pair of graphs.

been computed, it draws the matching connections and provides the user with a set of interaction functionalities for the visual analysis of the resulting drawing.

Criterion (C1) is motivated by several application scenarios that we had in mind during the design of the system. In these applications $G_{1}$ is often a graph whose entities represent geographic locations and therefore their position is either fixed or strongly constrained (examples are given in Sect. 4). About (C2), we focus on well recognized aesthetic criteria like number of crossings, number of bends, drawing area. Since the optimization of these criteria typically leads to an NP-hard problem, we propose some heuristics based on engineered versions of popular graph drawing algorithms, which are able to deal with the constraints of a one-to-many matched drawing. As an additional aesthetic criterion we require that $\left\langle\Gamma\left(G_{1}\right), \Gamma\left(G_{2}\right)\right\rangle$ is computed in such a way that the matching connections can be always drawn without intersecting the edges of $G_{2}$. When $G_{2}$ is a dense graph, $\Gamma\left(G_{2}\right)$ may have a high visual complexity, which makes it difficult to read the drawing at a whole, independently of the applied drawing strategy. This is the motivation for $(\mathbf{C 3})$.

\subsection{Drawing Algorithm}

Our drawing strategy for $\Gamma\left(G_{2}\right)$ combines different drawing conventions. We use orthogonal drawings for the layout of the rectangular regions $R(u)$ and their connections. Circular drawings are used to represent $G(M(u))$ inside $R(u)$. Finally, in order to simplify the visual complexity, we adopt a bundling operation for the edges connecting a vertex inside a region $R(u)$ to vertices outside $R(u)$; to avoid ambiguity, we use a "confluent-like" representation for these edges, as explained later. The algorithms used for the different drawing conventions have been engineered in order to deal with a certain number of constraints. In the following we describe in detail the steps performed by our drawing algorithm. We denote by $V_{i}$ and $E_{i}$ the set of vertices and edges of $G_{i}$, respectively $(i \in\{1,2\})$.

Step 1: Planarization. The goal of this step is to compute a suitable planar embedding of the graph consisting of "cluster vertices" and their interconnections, possibly replacing edge crossings with dummy vertices. More precisely, let $u_{1}, u_{2}, \ldots, u_{n}$ be the vertices 

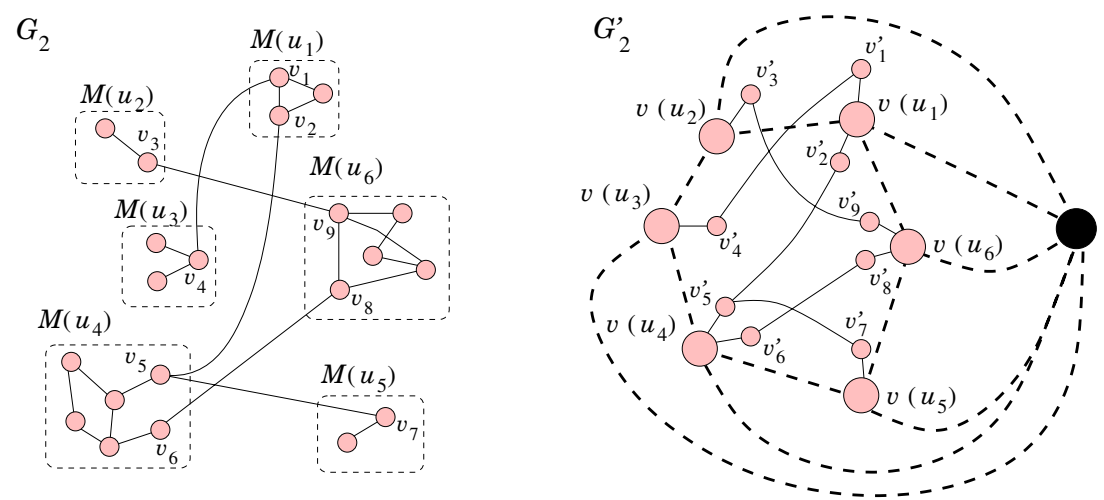

Fig. 3. (a) A graph $G_{2}$. (b) The graph $G_{2}^{\prime}$ used in Step 1 plus the wheel gadget (black node and dashed bold edges) adopted to guarantee (E1); the wheel gadget is removed at the end of Step 1.

of $G_{1}$ in the top-to-bottom order ${ }^{2}$ they appear in $\Gamma\left(G_{1}\right)$, and let $G_{2}^{\prime}$ be the graph obtained from $G_{2}$ by collapsing each cluster $M\left(u_{i}\right)$ into a single vertex $v\left(u_{i}\right)(1 \leq i \leq n)$, called a cluster vertex. In $G_{2}^{\prime}$ edges connecting vertices in the same cluster $M(u)$ disappear, while an edge connecting a vertex in $M\left(u_{i}\right)$ to a vertex in $M\left(u_{j}\right)(i \neq j)$ is transformed to a corresponding edge between $v\left(u_{i}\right)$ and $v\left(u_{j}\right)$. We aim at computing a planar embedding $\Psi$ of $G_{2}^{\prime}$ that satisfies the following two conditions: (E1) Cluster vertices $v\left(u_{1}\right), v\left(u_{2}\right), \ldots, v\left(u_{n}\right)$ appear counterclockwise in this order on the external face of $\Psi$; (E2) If $v \in M\left(u_{i}\right)$ in $G_{2}$ and if $e_{1}, \ldots, e_{k}$ are edges of $G_{2}$ incident to $v$, then the edges corresponding to $e_{1}, \ldots, e_{k}$ in $G_{2}^{\prime}$ appear consecutively (not necessarily in this order) around $v\left(u_{i}\right)$ in $\Psi$. Condition (E1) will guarantee Property (P3), i.e., the possibility of routing the matching connections without crossings among them; it also avoids crossings between matching edges and the edges of $G_{2}$. Condition (E2) makes it possible to simplify the links between the outside and the inside of each region $R\left(u_{i}\right)$ in the final drawing and to bundle these links as it will be explained in Step 3. To force (E2) we further transform $G_{2}^{\prime}$ by attaching to $v\left(u_{i}\right)$ a vertex $v^{\prime}$ for each vertex $v \in M\left(u_{i}\right)$ connected to vertices outside $M\left(u_{i}\right)$, and by replacing the edges $e_{1}, \ldots, e_{k}$ that are incident to $v$ with corresponding edges $e_{1}^{\prime}, \ldots, e_{k}^{\prime}$ connected to $v^{\prime}$. Vertex $v^{\prime}$ is called the image of $v$.

On $G_{2}^{\prime}$ we apply a standard planarization algorithm based on first extracting a maximal planar subgraph and then on iteratively reinserting the discarded edges by computing shortest paths in the dual graph and by replacing edge crossings with dummy vertices [10]. To force (E1), we use a "wheel gadget" of uncrossable edges that will be removed at the end of the planarization phase. Figure 3 shows an example of a graph $G_{2}^{\prime}$ and the wheel gadget used to guarantee (E1).

Notice that, quadratic and linear-time algorithms for planarity testing and edge reinsertion within the above described embedding constraints have been also proposed in $[1,17]$. Our planarization phase takes $O\left(\left|E_{2}\right|\left(c+\left|V_{2}\right|\right) \log \left(c+\left|V_{2}\right|\right)\right)$ time, where $c$ is the number of edge crossings in the final embedding of $G_{2}^{\prime}$.

${ }^{2}$ If $u_{i}$ and $u_{j}$ have the same $y$-coordinate, they are ordered from right to left. 
Step 2: Orthogonalization and Compaction. Once a planar embedding $\Psi$ of $G_{2}^{\prime}$ (with possible cross vertices) has been found, an orthogonal drawing of $G_{2}^{\prime}$ that preserves $\Psi$ is computed. The basic idea is to use an orthogonal drawing algorithm that deals with arbitrary vertex degree and that allows for vertex size customization. Indeed, we want that $v\left(u_{i}\right)$ is drawn as a box big enough to host all vertices of $M\left(u_{i}\right)$. To this aim, the system uses the network flow based drawing algorithm described in [9], which represents a good heuristic both in terms of bend minimization and in terms of area drawing compaction. Denoted by $B\left(v\left(u_{i}\right)\right)$ the box representing vertex $v\left(u_{i}\right)$, we draw $B\left(v\left(u_{i}\right)\right)$ as a square of a certain size $r_{i}$. In the final drawing we place a circle of radius $\rho_{i}$ inside $B\left(v\left(u_{i}\right)\right)$ and equi-distribute along its perimeter the vertices of $M\left(u_{i}\right)$. To determine $\rho_{i}$, we fix a minimum distance $\delta$ we want to guarantee between any two vertices of $M\left(u_{i}\right)$ and we set $\rho_{i}=\delta \cdot\left|M\left(u_{i}\right)\right| / 2 \pi$. We choose $r_{i}$ to be larger enough than $\rho_{i}$ so that it is possible to route the edges connecting vertices inside $B\left(v\left(u_{i}\right)\right)$ with the outside. Each square $B\left(v\left(u_{i}\right)\right)$ will correspond to region $R\left(u_{i}\right)$ in the final drawing. Also, in order to guarantee the properties of a one-to-many matched drawing, we add a certain number of constraints as described below.

If one wants to compute a strong one-to-many matched drawing, then all vertices $v\left(u_{1}\right), v\left(u_{2}\right), \ldots, v\left(u_{n}\right)$ are temporarily connected in this order to form a simple cycle $C$ that becomes the new boundary for the external face. Then the following angle and bend constraints on the vertices and edges of $C$ are imposed: Each edge of $C$ connecting $v\left(u_{i}\right)$ to $v\left(u_{i+1}\right)(1 \leq i \leq n-1)$ is constrained to be straight-line in the drawing, while the edge of $C$ connecting $v\left(u_{n}\right)$ to $v\left(u_{1}\right)$ is constrained to turn always in the left direction while moving from $v\left(u_{n}\right)$ to $v\left(u_{1}\right)$. Each angle formed at a vertex $v\left(u_{i}\right)$ on the external face is set to be of 180 degrees. These constraints guarantee that $v\left(u_{1}\right), v\left(u_{2}\right), \ldots, v\left(u_{n}\right)$ are encountered from top-to-bottom in the final drawing and that they are all visible from left. Once a drawing has been computed the edges of $C$ are removed. If one wants to compute a (not necessarily strong) one-to-many matched drawing, then we still construct cycle $C$, but we only impose the constraint that the edges of $C$ turn in the left direction or go straight while moving along $C$ counterclockwise. Finally, in order to correctly perform the next step (i.e., the edge bundling operation), we also require that for each image vertex $v^{\prime}$ attached to a vertex $v\left(u_{i}\right)$, there is no other edge incident to $v^{\prime}$ from the same direction of edge $\left(v\left(u_{i}\right), v^{\prime}\right)$.

All the orthogonalization constraints described above are translated into constraints on the flow network of the algorithm in [9]. The orthogonalization and compaction phases take $O\left(\left(\left|V_{1}\right|\left|V_{2}\right|+c\right)^{2} \log \left(\left|V_{1}\right|\left|V_{2}\right|+c\right)\right)$ time, where $c$ is still the number of cross vertices in the embedding $\Psi$.

Step 3: Edge Bundling. This step removes each image vertex $v^{\prime}$ and creates in its place a "confluent-like" structure for the edges incident to $v^{\prime}$. Namely, let $v$ be the vertex of the original graph that has $v^{\prime}$ as its image and let $M\left(u_{i}\right)$ be the cluster that contains $v$. Let $e_{1}^{\prime}, \ldots, e_{k}^{\prime}$ be the edges incident to $v^{\prime}$ other than edge $\left(v^{\prime}, v\left(u_{i}\right)\right)$. We want that $v^{\prime}$ is no longer present in the final drawing and that the edges $e_{1}^{\prime}, \ldots, e_{k}^{\prime}$ are replaced by the edges $e_{1}, e_{2}, \ldots, e_{k}$ that were originally connected to $v$. To simplify the final drawing however, we bundle the edges $e_{1}, e_{2}, \ldots, e_{k}$ from $v$ to $v^{\prime}$; this edge bundle follows the drawing of $e$ from the boundary of $R\left(u_{i}\right)$ to $v^{\prime}$ and then it divides in $k$ branches at $v^{\prime}$ using splines, as shown in Fig. 4(a). It is important to remark that the 


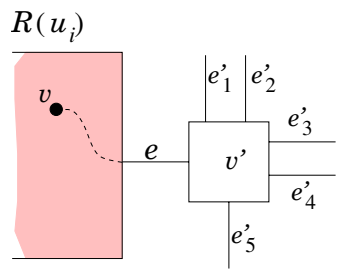

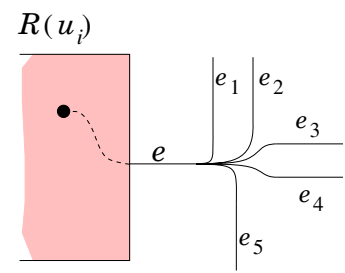

(a)

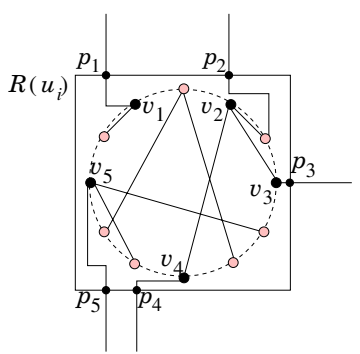

(b)

Fig. 4. (a) Illustration of Step 3. The image vertex $v^{\prime}$ is removed and its incident edges are replaced by a "confluent-like" structure. The dashed curve is the part of edge bundle that will be drawn in Step 4. (b) Illustration of Step 4. The black vertices inside $R\left(u_{i}\right)$ denote the vertices whose relative circular ordering is fixed according to their corresponding external connections.

edge bundling operation guarantees that for each vertex $v$ inside a region $R\left(u_{i}\right)$ there will be at most one link (a bundle of edges) incident to $v$ from the outside of $R\left(u_{i}\right)$. Since these links must be routed around the circular drawing representing $G\left(M\left(u_{i}\right)\right.$ ), this property strongly simplifies the visual complexity introduced by these connections. The edge bundling step takes $O\left(\left|E_{2}\right|\right)$ time.

Step 4: Circular Drawing Computation. At the end of the previous step, we have a partial drawing of $G_{2}$ such that for each cluster vertex $v\left(u_{i}\right)$ there is a corresponding rectangular region $R\left(u_{i}\right)$ and some edges incident to the boundary of $R\left(u_{i}\right)$ at certain points $p_{1}, p_{2}, \ldots, p_{k}$. To complete the drawing of $G_{2}$ we construct a circular drawing for each $G\left(M\left(u_{i}\right)\right)$, and then connect $p_{j}$ to its corresponding vertex $v_{j}$ of $M\left(u_{i}\right)(1 \leq j \leq k)$. See Fig. 4(b) for an illustration. In order to avoid crossings between links $\left(p_{j}, v_{j}\right)$, we force the circular order of vertices $v_{j}$ to be consistent with the circular order of points $p_{1}, p_{2}, \ldots, p_{k}$ around $R\left(u_{i}\right)$, i.e., if $p_{1}, p_{2}, \ldots, p_{k}$ occur clockwise in this order around $R\left(u_{i}\right)$ then we force $v_{1}, v_{2}, \ldots, v_{k}$ to occur clockwise in this order in the circular drawing. Conversely, all vertices of $M\left(u_{i}\right)$ distinct from $v_{j}(1 \leq j \leq k)$ can be placed everywhere in the circular ordering (these vertices are not connected to vertices outside $R\left(u_{i}\right)$ ). In other words, if $V_{f i x}=\left\{v_{1}, v_{2}, \ldots, v_{k}\right\}$ and $V_{\text {free }}=M\left(u_{i}\right) \backslash V_{f i x}$, we want to find a "good" circular order for the vertices of $M\left(u_{i}\right)$ such that the relative order of the vertices of $V_{f i x}$ is fixed; our goal is the minimization of the number of edge crossings, which is however an NP-Hard problem [18]. To solve it, we designed a variation of the heuristic described by Baur and Brandes [5], which has been experimentally shown to produce better results in terms of crossing reduction than previous heuristics for computing circular drawings, and that has been successfully adopted for the layout of two-level networks that are similar to the clustered structure of $G_{2}$ [6]. We also recall that faster but less effective circular drawing algorithms in terms of edge crossings have been described in [20]. The heuristic by Baur and Brandes computes an ordering of the vertices on a straight line $\ell$, assuming that all edges are drawn on the same half-plane determined by $\ell$. In terms of edge crossings this model is equivalent to place the vertices on a circle and to draw the edges as straight-line 
segments. At the end of this placement greedy heuristic, a post-processing step, called circular sifting is applied to further reduce the number of edge crossings if possible. The idea is to iteratively swapping a vertex with its successor vertex in the linear order on $\ell$ and recording the change in crossing count; the vertex is then placed in the position that corresponds to its local optimal. Denoted by $n$ and $m$ the number of vertices and the number of edges of the input graph, respectively, the placement greedy heuristic can be performed in $O((n+m) \log n)$ time, while repositioning each vertex once in the circular sifting phase can be done in $O(\mathrm{~nm})$ time (see [5]).

Our variation of the algorithm in [5] works as follows. The placement greedy heuristic performs analogously to the one of Baur and Brandes, but it assumes that the vertices of $V_{f i x}$ are already placed on $\ell$ in a preassigned order; therefore the placement decisions are restricted to the vertices of $V_{\text {free }}$. The circular sifting phase is modified so that swaps between vertices both belonging to $V_{f i x}$ are not allowed. Once the circular ordering of the vertices of $M\left(u_{i}\right)$ has been computed, the algorithm equi-distributes these vertices on a circle inside $R\left(u_{i}\right)$ and rotates this circle in order to reduce the total length of the connections $\left(p_{j}, v_{j}\right)(1 \leq j \leq k)$, which are routed as polygonal chains of vertical and horizontal segments. The circular drawing computation over all cluster vertices takes $O\left(\left|V_{1}\right|\left(\left(\left|V_{2}\right|+\left|E_{2}\right|\right) \log \left|V_{2}\right|+\left|V_{2}\right|\left|E_{2}\right|\right)\right)$ time (recall that $\left|V_{1}\right|$ corresponds to the number of cluster vertices).

Step 5: Drawing of Matching Edges. This step is simply performed by routing the matching edges as polygonal chains from the location of a vertex $u_{i}$ of $\Gamma\left(G_{1}\right)$ to the boundary of the corresponding region $R\left(u_{i}\right)$ in $\Gamma\left(G_{2}\right)$. Since the circular ordering of the regions on the external face of $\Gamma\left(G_{2}\right)$ is consistent with the top-down ordering of the corresponding vertices in $\Gamma\left(G_{1}\right)$, this can be done without crossing between matching edges. Also, in a strong one-to-many matched drawing, each matching edge can be routed with at most two bends.

Time Complexity. The next theorem summarizes the discussion about the drawing algorithm implemented in $M O M$. To simplify the time complexity of this algorithm, the statement of the theorem assumes that $\left|V_{1}\right|$ is bounded by a constant. This appears as a reasonable assumption if $\left|V_{1}\right| \ll\left|V_{2}\right|$.

Theorem 1. Let $\left\langle G_{1}, G_{2}\right\rangle$ be a pair of one-to-many matched graphs such that $G_{1}=$ $\left(V_{1}, E_{1}\right)$ and $G_{2}=\left(V_{2}, E_{2}\right)$. Let $\Gamma\left(G_{1}\right)$ be any drawing of $G_{1}$. There exists a polynomial-time algorithm that computes a one-to-many matched drawing $\left\langle\Gamma\left(G_{1}\right), \Gamma\left(G_{2}\right)\right\rangle$ (either in the strong or in the non-strong model) with the additional property that the matching edges can be drawn without intersecting any vertex and edge of $\Gamma\left(G_{2}\right)$. Also, if $\left|V_{1}\right|$ is bounded by a constant, and denoted by $N$ the number $N=\left|V_{2}\right|+c$, where $c$ is the number of inter-cluster edge crossings in $\Gamma\left(G_{2}\right)$, then the time complexity of the drawing algorithm is: $O\left(\left(\left|E_{2}\right| N+N^{2}\right) \log N\right)$.

\subsection{Interaction Functionalities}

In order to facilitate the visual analysis of the computed one-to-many matched drawings, we equipped our system with a certain number of interaction functionalities, other than conventional zooming and translation primitives. We briefly describe them in the following. 
Cluster Expansion/Contraction: By default, all cluster regions $R(u)$ in $\Gamma\left(G_{2}\right)$ are expanded, i.e., the whole subgraph inside each $R(u)$ is displayed by the system. In order to compact the drawing and/or to hide some details, the user can decide to contract a certain number of clusters by simply clicking on them. A cluster contraction redraws the cluster as a small box and hides its content. Every cluster can be expanded or contracted an infinite number of times without any restriction. After a cluster expansion/contraction, the drawing is automatically re-compacted by the system, but the orthogonal shape of the drawing remains unchanged, so to avoid that the user mental map is lost. Contracting clusters can be useful to get an overview of the inter-cluster relations before analyzing the intra-cluster ones.

Cluster Filtering: If the user is interested in focusing on some of the clusters, she can select them and hide the remaining clusters and their connections. After such an operation, the user can also decide to re-compact the remaining part of the drawing to save space if possible. When the drawing of $\Gamma\left(G_{2}\right)$ has many clusters and/or many inter-cluster links, the cluster filtering primitive can help to explore the graph structure portion by portion.

Edge Filtering: Our system allows the representation of edge weighted graphs. This means that a weight can be assigned to each edge of $G_{1}$ and of $G_{2}$. When a graph is too dense, the user can sparsify the links by setting an edge visibility threshold. All links having the weight below the given threshold are not shown by the system. Again, the drawing is re-compacted if required.

Edge/Vertex Highlighting: Moving the mouse over a certain vertex or cluster region, the user can decide to highlight all edges incident to that vertex or to that cluster region. A tooltip with information about the selected vertex is also displayed. This helps to get local information on the drawing. Furthermore, moving the mouse over an edge, a tooltip that displays the labels of its end-vertices is shown. This helps when just a portion of the selected edge fits in the current view.

\section{Application Examples}

One-To-Many matched graphs occur in several applications contexts. Here we briefly present an example on social network analysis. Another application example on Web search clustering engines is described in [12].

Our example focuses on the co-authorship network of the last Symposium on Graph Drawing, GD 2007. $G_{1}$ is the graph having European countries as vertices and edges between countries that cooperated in co-authoring some papers. Each edge has a weight equal to the number of papers resulting from the cooperation of the connected countries. The drawing $\Gamma\left(G_{1}\right)$ is a simple straight-line drawing, where each vertex is placed at a fixed location on a geographic map. Graph $G_{2}$ represents authors and their cooperations in the articles. Figure 5 shows a one-to-many matched drawing in the strong model. The drawing gives an overview of the network structure, which reveals the number of contributing authors for each country and a relevant level of cooperation among the different countries. Looking inside a country, it is possible to see its different subcommunities. For example, it is easy to recognize two sub-communities in Greece, in Italy, and in Czech Republic, several communities in Germany, and one big community 


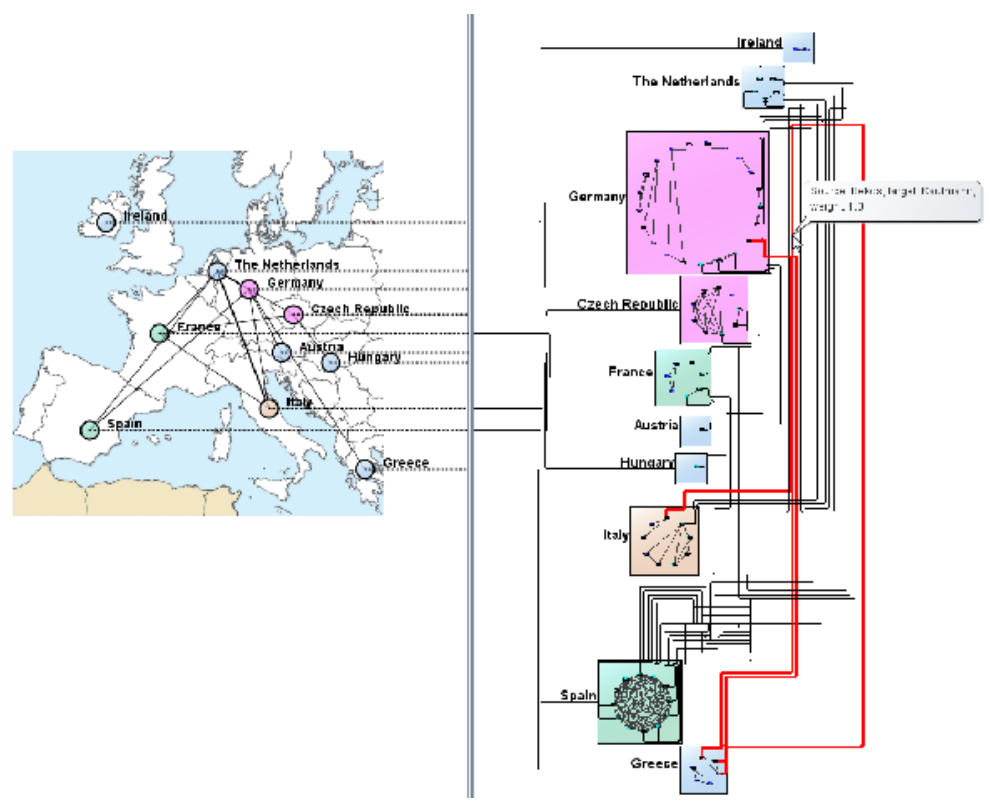

Fig. 5. A one-to-many matched drawing showing the European co-authorship network of GD 2007

in Spain. Selecting an author in a country, all her connections with other authors are highlighted by the system. In the figure, author "Kaufmann" inside Germany is selected, and the system highlights (in bold red color) his connections with other authors, three in Greece and one in Italy. Moving the mouse over one of the bold red edges, it is displayed a tooltip that reports the labels of its end-vertices. Figure 6 shows an example of edge and vertex filtering on the previous drawing, which makes it easier to focus on specific relationships. Namely, the edges of $\Gamma\left(G_{1}\right)$ has been filtered so that only those edges with a weight greater than 1 are shown. The vertices of $\Gamma\left(G_{2}\right)$ have been filtered in such a way that only the countries having some incident links in $\Gamma\left(G_{1}\right)$ are shown (i.e., Germany, Italy, and The Netherlands). Then, cluster Germany has been contracted to focus on the interplay between Italy and The Netherlands. After the vertex filtering and contraction operations, $\Gamma\left(G_{2}\right)$ is recomputed so to become more compact without destroying the user's mental map. In the figure, the connections of author "Meijer" are highlighted in bold red.

\section{System Performances}

We have tested our system in order to measure its performances. Our main goal was to measure the running time and some important aesthetic requirements, like number of crossings, number of bends, drawing area, and aspect ratio (width/height). We compared the algorithm for strong one-to-many matched drawings against the algorithm for non-strong one-to-many matched drawings, so to understand the trade-off between the 


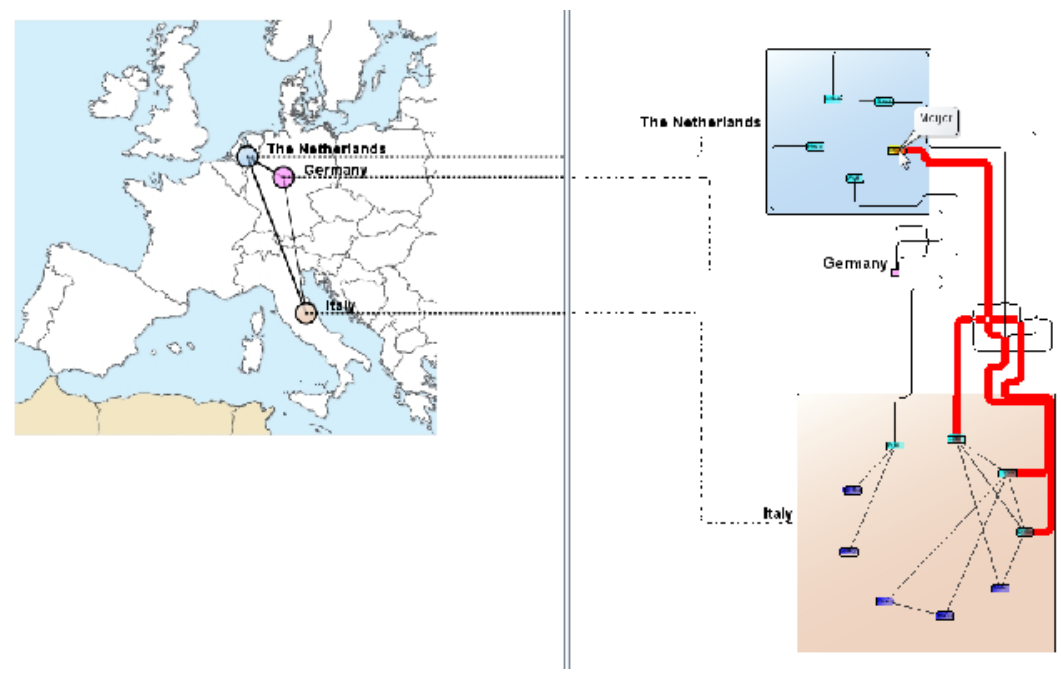

Fig. 6. The same one-to-many matched graphs of Fig. 5 after some edge and vertex filtering

results of the two algorithms. A strong drawing greatly helps in the readability of the matching between $G_{1}$ and $G_{2}$, but we expect that a strong drawing has worst values for some aesthetics (e.g., aspect ratio and number of bends) than for a non-strong drawing.

The focus is on the drawing of $G_{2}$, because we are assuming that a drawing $\Gamma\left(G_{1}\right)$ is given as part of the input or that it is computed with some classical drawing algorithm. For the experiments we used a test suite of instances for $G_{2}$, with given number of cluster vertices. We generated 240 graphs in total, 5 graphs for each sample. A sample is obtained by fixing number of vertices, number of clusters, and density (number of edges/number of vertices). The number of vertices is a value in the set $\{100,400,700,1000\}$, the number of clusters is a value in $\{5,10,15,20\}$, and the density is a value in $\{1,1.5,2\}$. Each graph was generated at random, by assuming that $10 \%$ of the edges are inter-cluster edges and that $90 \%$ of the edges are intra-cluster edges. The experiments have been executed under the Windows 2003 server OS, on an Intel Pentium IV with $3.0 \mathrm{GHz}$ and $2 \mathrm{~GB}$ of RAM.

The charts of the experimental results are omitted for reasons of space and can be found in [12]. As for the running time, the computation of strong drawings is slightly slower than for non-strong drawings (in the average, it requires about $10 \%$ more). In general, both types of computations take a few seconds for graphs up to 400 vertices and low density values. Graphs with the highest density and 700 vertices are computed in a few minutes, while the computations may require up to 30 minutes for the hardest instances of our test suite, i.e., graphs with 1000 vertices and density 2. About the area and the aspect ratio, since in a strong one-to-many matched drawing every two cluster regions are constrained to stay one below the other, strong drawings have a worst aspect ratio but smaller area than non-strong drawings, which have aspect ratio close to 1 . About the number of bends, strong drawings present in the average $11-12 \%$ 
of bends more than non-strong drawings, which are caused by their greater number of constraints. Finally, as already observed, the number of crossings is independent of the two drawing algorithms, and as expected it rapidly increases with the graph density.

\section{References}

1. GDToolkit: Graph drawing toolkit, http: / /www. dia.uniroma3.it/ gdt/

2. IEEE Symposium on Information Visualization (InfoVis 2007), October 28-30. IEEE Computer Society, Sacramento (2007)

3. Hong, S.-H., Nishizeki, T., Quan, W. (eds.): GD 2007. LNCS, vol. 4875. Springer, Heidelberg (2008)

4. IEEE VGTC Pacific Visualization Symposium 2008 (PacificVis 2008), Kyoto, Japan, March 4-7. IEEE Computer Society, Los Alamitos (2008)

5. Baur, M., Brandes, U.: Crossing reduction in circular layouts. In: Hromkovič, J., Nagl, M., Westfechtel, B. (eds.) WG 2004. LNCS, vol. 3353, pp. 332-343. Springer, Heidelberg (2004)

6. Baur, M., Brandes, U.: Multi-circular layout of micro/Macro graphs. In: Hong, S.-H., Nishizeki, T., Quan, W. (eds.) GD 2007. LNCS, vol. 4875, pp. 255-267. Springer, Heidelberg (2008)

7. Braß, P., Cenek, E., Duncan, C.A., Efrat, A., Erten, C., Ismailescu, D., Kobourov, S.G., Lubiw, A., Mitchell, J.S.B.: On simultaneous planar graph embeddings. Comput. Geom. Theory and Appl. 36(2), 117-130 (2007)

8. Collins, C., Carpendale, M.S.T.: Vislink: Revealing relationships amongst visualizations. IEEE Trans. Vis. Comput. Graph. 13(6), 1192-1199 (2007)

9. Di Battista, G., Didimo, W., Patrignani, M., Pizzonia, M.: Orthogonal and quasi-upward drawings with vertices of prescribed size. In: Kratochvíl, J. (ed.) GD 1999. LNCS, vol. 1731, pp. 297-310. Springer, Heidelberg (1999)

10. Di Battista, G., Eades, P., Tamassia, R., Tollis, I.G.: Graph Drawing. Prentice-Hall, Englewood Cliffs (1999)

11. Di Giacomo, E., Didimo, W., van Kreveld, M., Liotta, G., Speckmann, B.: Matched drawings of planar graphs. In: Hong, S.-H., Nishizeki, T., Quan, W. (eds.) GD 2007. LNCS, vol. 4875, pp. 183-194. Springer, Heidelberg (2008)

12. Di Giacomo, E., Didimo, W., Palladino, P., Liotta, G.: Visual analysis of one-to-many matched graphs. Tech. rep, RT-003-08, DIEI - Università di Perugia, Italy (2008)

13. Di Giacomo, E., Liotta, G.: Simultaneous embedding of outerplanar graphs, paths, and cycles. Intern. Journ. of Comput. Geom. and Appl. 17(2), 139-160 (2007)

14. Erten, C., Harding, P.J., Kobourov, S.G., Wampler, K., Yee, G.V.: Graphael: Graph animations with evolving layouts. In: Liotta, G. (ed.) GD 2003. LNCS, vol. 2912, pp. 98-110. Springer, Heidelberg (2004)

15. Erten, C., Kobourov, S.G.: Simultaneous embedding of a planar graph and its dual on the grid. Theory Comput. Syst. 38(3), 313-327 (2005)

16. Erten, C., Kobourov, S.G., Le, V., Navabi, A.: Simultaneous graph drawing: Layout algorithms and visualization schemes. Journ. Graph Alg. and Appl. 9(1), 165-182 (2005)

17. Gutwenger, C., Klein, K., Mutzel, P.: Planarity testing and optimal edge insertion with embedding constraints. Journ. of Graph Alg. and Appl. 12(1), 73-95 (2008)

18. Masuda, S., Kashiwabara, T., Nakajima, K., Fujisawa, T.: On the NP-completeness of a computer network layout problem. In: 20th IEEE Int. Symposium on Circuits and Systems, pp. 292-295 (1987)

19. Shneiderman, B., Aris, A.: Network visualization by semantic substrates. IEEE Trans. Vis. Comput. Graph. 12(5), 733-740 (2006)

20. Six, J.M., Tollis, I.G.: A framework and algorithms for circular drawings of graphs. Journ. of Discr. Alg. 4(1), 25-50 (2006) 